\title{
Alterations in dorsal and ventral posterior cingulate connectivity in APOE $\varepsilon 4$ carriers at risk of Alzheimer's disease
}

Rebecca Kerestes, Pramit M. Phal, Chris Steward, Bradford A. Moffat, Simon Salinas, Kay L. Cox, Kathryn A. Ellis, Elizabeth V. Cyarto, David Ames, Ralph N. Martins, Colin L. Masters, Christopher C. Rowe, Matthew J. Sharman, Olivier Salvado, Cassandra Szoeke, Michelle Lai, Nicola T. Lautenschlager ${ }^{*}$ and Patricia M. Desmond ${ }^{*}$

\section{Background}

Recent evidence suggests that exercise plays a role in cognition and that the posterior cingulate cortex (PCC) can be divided into dorsal and ventral subregions based on distinct connectivity patterns.

\section{Aims}

To examine the effect of physical activity and division of the PCC on brain functional connectivity measures in subjective memory complainers (SMC) carrying the epsilon 4 allele of apolipoprotein E (APOE $\varepsilon 4$ ) allele.

\section{Method}

Participants were 22 SMC carrying the APOE $\varepsilon 4$ allele $(\varepsilon 4+$; mean age 72.18 years) and 58 SMC non-carriers $(\varepsilon 4-$; mean age 72.79 years). Connectivity of four dorsal and ventral seeds was examined. Relationships between PCC connectivity and physical activity measures were explored.

\section{Results}

$\varepsilon 4+$ individuals showed increased connectivity between the dorsal PCC and dorsolateral prefrontal cortex, and the ventral PCC and supplementary motor area (SMA). Greater levels of physical activity correlated with the magnitude of ventral PCCSMA connectivity.

\section{Conclusions}

The results provide the first evidence that $\varepsilon 4+$ individuals at increased risk of cognitive decline show distinct alterations in dorsal and ventral PCC functional connectivity.

\section{Declaration of interest}

D.A. has served on scientific advisory boards for Novartis, Eli Lilly, Janssen, Prana and Pfizer, and as Editor-in-Chief for International Psychogeriatrics; received speaker honoraria from Pfizer and Lundbeck, and research support from Eli Lilly, GlaxoSmithKline, Forest Laboratories, Novartis, and CSIRO. C.L.M. has received consulting fees from Eli Lilly and Prana Biotechnology, and has stock ownership in Prana Biotechnology. C.C.R. has received consultancy payments from Roche and Piramal, and research support from Avid Radiopharmaceuticals, Eli Lilly, GE Healthcare, Piramal and Navidea for amyloid imaging. C.S. has provided clinical consultancy and been on scientific advisory committees for the Australian CSIRO, Alzheimer's Australia, University of Melbourne and other relationships, which are subject to confidentiality clauses; she has been a named Chief Investigator on investigator-driven collaborative research projects in partnership with Pfizer, Merck, Piramal, Bayer and GE Healthcare. Her research programme has received support from the National Health and Medical Research Council Alzheimer's Association, Collier Trust, Scobie and Claire McKinnon Foundation, JO and JR Wicking Trust, Shepherd Foundation, Brain Foundation, Mason Foundation, Ramaciotti Foundation, Alzheimer's Australia and the Royal Australian College of Physicians.

\section{Copyright and usage}

(c) The Royal College of Psychiatrists 2015. This is an open access article distributed under the terms of the creative commons Non-Commercial, No Derivatives (CC BY-NC-ND) licence.
The epsilon 4 allele of apolipoprotein $\mathrm{E}$ (APOE $\varepsilon 4$ ) is the most well-established genetic susceptibility factor for Alzheimer's disease pathology. ${ }^{1}$ Heterozygous APOE $\varepsilon 4$ carriers are at twofold to threefold increased risk of Alzheimer's disease and the risk is $\sim 12$-fold for homozygous carriers. ${ }^{1}$ Accumulating evidence implicates APOE $\varepsilon 4$ with alterations in brain function and structure in otherwise healthy young, middle-aged and older carriers of the APOE $\varepsilon 4$ allele. $^{2-4}$

Resting-state functional magnetic resonance imaging (fMRI) is a powerful tool for examining the large-scale organisation of functional connectivity of brain networks in healthy and clinical populations. ${ }^{5}$ One of the most well-studied intrinsic resting-state networks is the default mode network (DMN). The DMN is a set of brain regions comprising the posterior cingulate, the medial frontal cortex, lateral and inferior parietal cortices, the hippocampus and inferior temporal cortex. ${ }^{6,7}$ Regions of the DMN are more 'active' during rest and are implicated in self-referential and

These authors contributed equally to this work. memory-encoding processes (e.g. recalling the past). ${ }^{6,7}$ Alterations in DMN connectivity have been found in individuals at risk of Alzheimer's disease including individuals with mild cognitive impairment ${ }^{8,9}$ and subjective memory complainers (SMC), ${ }^{10}$ consistent with the notion that Alzheimer's disease pathology forms preferentially in regions that represent the DMN. ${ }^{7}$ However, despite evidence that the APOE $\varepsilon 4$ allele modulates DMN connectivity in healthy individuals, ${ }^{11}$ little is known about how the APOE $\varepsilon 4$ allele modulates DMN connectivity in individuals at risk of cognitive decline and progression to Alzheimer's disease.

The posterior cingulate cortex (PCC) has a key role in the $\mathrm{DMN}$ and is heavily implicated in memory-related processes. Metabolic, ${ }^{12}$ functional ${ }^{13}$ and structural abnormalities ${ }^{14}$ of the PCC have long been implicated in the pathophysiology of Alzheimer's disease. ${ }^{15}$ Recently, researchers have proposed topographic divisions of the PCC, consisting of dorsal and ventral subregions based on their distinct functional connectivity patterns with other intrinsic resting-state networks. ${ }^{15-17}$ In line with this view, the ventral subdivision of the PCC is connected primarily to other DMN regions to support internally directed thought during 
the resting state, but deactivates and is anticorrelated with dorsal attention and frontoparietal control network regions when attention is externally focused during cognitive tasks. Similarly, the dorsal PCC also shows rapid deactivation during simple cognitive tasks when attentional demands are high. However, in contrast to the ventral PCC, the dorsal PCC displays a more complex, dynamic pattern of connectivity to regions spanning multiple networks during rest. These include parts of the frontoparietal network, sensorimotor network regions, as well as lateral and medial temporal cortices. ${ }^{16,17}$ In light of these findings, it has been suggested that the PCC should not be treated as having a unitary function and rather that the transitional pattern of dorsal PCC connectivity during rest may serve to 'link' brain networks that are functionally distinct, to achieve efficient cognitive function. ${ }^{15}$

The primary aim of our study was to assess the association between APOE $\varepsilon 4$ and functional connectivity of the dorsal and ventral PCC in a population at risk of cognitive decline and progressing to Alzheimer's disease, namely individuals with SMC with and without mild cognitive changes. Systematically examining dorsal and ventral subdivisions of the PCC is more sensitive to detecting subtle changes in functional connectivity patterns of the PCC. Given that this was the first study to use this approach in a population at risk of Alzheimer's disease, the results of the study have important implications for understanding neurodegenerative processes at the early stages of cognitive decline. We hypothesised that APOE $\varepsilon 4$ carriers would show reduced functional connectivity of the dorsal and ventral PCC to predominantly frontoparietal regions and medial temporal cortex, compared with non-carriers. Furthermore, our study population participates in a randomised controlled trial (RCT) investigating the impact of physical activity on the progression of white matter disease. ${ }^{18}$ There is accumulating evidence of physical activity-induced changes in functional connectivity of large-scale brain networks including frontoparietal, sensorimotor and DMN regions, in healthy aging adults. ${ }^{19,20}$ Such changes may be expected to preferentially occur in regions that are affected by age-related cognitive decline including the PCC, and be modulated as a function of genetic vulnerability to Alzheimer's disease. Therefore, a secondary aim of this study was to examine the association between multiple independent measures of physical activity measured at baseline, and resting-state PCC connectivity in APOE $\varepsilon 4$ carriers and noncarriers. Based on the available literature, we hypothesised that higher levels of baseline physical activity would be associated with increased PCC connectivity with frontoparietal and sensorimotor network regions.

\section{Method}

\section{Participants}

Twenty-two individuals carrying at least one copy of the APOE $\varepsilon 4$ gene (referred to as APOE $\varepsilon 4+$; mean age 72.18 years, s.d.=5.79) and 58 age-, gender- and education-matched non-carriers (APOE $\varepsilon 4-$; mean age 72.79 years, s.d. $=5.69$ ) were recruited from an ongoing RCT assessing the effects of exercise on white matter disease progression ${ }^{18}$ (Table 1). All data reported herein are crosssectional and were measured at baseline before commencement of intervention. Each participant's MRI data included here satisfied our stringent imaging quality control criteria (see below).

Of the 22 APOE $\varepsilon 4+$ SMC individuals, 9 had accompanying mild cognitive changes. In the APOE $\varepsilon 4$ - group, 15 SMC individuals had accompanying mild cognitive changes. To be included in the study, participants had to meet the following criteria to be designated as an SMC: answer 'yes' to the following question: 'Do you have any difficulty with your memory?' yet score in the range of normal control groups for their age and gender on the cognitive battery of the Consortium to Establish a Registry for Alzheimer's Disease (CERAD) ${ }^{21}$ In addition, the presence of accompanying mild cognitive impairments was assessed using the following subjective and objective measures: (a) subjective memory complaint, (b) evidence of impairment on objective tasks (measured with a score on the CERAD lower than -1.5 s.d. or more compared with the normal control groups on any of the CERAD subtests, and (c) preserved activities of daily living, and only minimal impairment in complex instrumental functions. All participants did not have dementia. Details regarding demographic measures, physical activity measures, as well as inclusion/exclusion criteria can be found in the supplementary material. All participants provided written informed consent after a full description of the Aibl Active trial protocol was given to them. The Aibl Active trial (Trial registration number ACTRN12611000612910) was approved by the Melbourne Health Human Research Ethics Committee (HREC 2011.014) and is fully compliant with the guidelines of the World Medical Association Declaration of Helsinki.

\section{APOE genotyping}

APOE genotype was determined using polymerase chain reaction amplification and restriction enzyme digestions by the method previously described ${ }^{22}$ (see supplementary material). Observed genotypic frequencies were as follows: APOE $\varepsilon 2 / \mathrm{APOE}$ $\varepsilon 2(n=2)$, APOE $\varepsilon 2 / \mathrm{APOE} \varepsilon 4(n=3)$, APOE $\varepsilon 3 / \mathrm{APOE} \varepsilon 2(n=7)$, APOE $\varepsilon 3 / \mathrm{APOE} \varepsilon 3(n=49)$, APOE $\varepsilon 3 / \mathrm{APOE} \varepsilon 4 \quad(n=18)$, and APOE $\varepsilon 4 / \mathrm{APOE} \varepsilon 4(n=1)$.

Table 1 Demographic information, memory scores and volumetric data for APOE $\varepsilon 4+$ and APOE $\varepsilon 4$ - participants

\begin{tabular}{|c|c|c|c|c|}
\hline & $\begin{array}{c}\text { APOE } \varepsilon 4- \\
n=58\end{array}$ & $\begin{array}{c}\text { APOE } \varepsilon 4+ \\
n=22\end{array}$ & Statistics & $\begin{array}{c}p \\
\text { (two-tailed) }\end{array}$ \\
\hline Age, years: mean (s.d.) & $72.79(5.69)$ & $72.18(5.79)$ & $t(78)=0.42$ & 0.67 \\
\hline Female, \% (n) & $53(31)$ & $63(14)$ & $\chi^{2}(1)=0.67$ & 0.41 \\
\hline Education, years: mean (s.d.) & $14.34(3.54)$ & $14.45(4.47)$ & $t(78)=0.11$ & 0.15 \\
\hline MMSE, mean (s.d.) & $28.67(1.71)$ & $28.77(1.37)$ & $t(78)=0.24$ & 0.80 \\
\hline ADAS-Cog 11 score, mean (s.d.) & $7.27(3.92)$ & $7.18(3.43)$ & $t(78)=0.09$ & 0.40 \\
\hline PA, no. steps per week ${ }^{\mathrm{a}}$ & 50436 & 60329 & $t(77)=1.80$ & 0.08 \\
\hline PA, no. minutes MIE per week: mean (s.d.) & $143(117)$ & $187(109)$ & $t(78)=1.43$ & 0.15 \\
\hline PA, distance 6-minute walk test: mean (s.d.) & $487.65(91.83)$ & $526.09(89.53)$ & $t(78)=1.68$ & 0.09 \\
\hline Whole brain volume, mean (s.d. $)^{b}$ & $69(0.05)$ & $68(0.06)$ & $t(78)=0.77$ & 0.79 \\
\hline \multicolumn{5}{|c|}{$\begin{array}{l}\text { APOE } \varepsilon+\text {, apolipoprotein } 4 \text { allele carrier; APOE } \varepsilon 4-\text {, apolipoprotein } 4 \text { allele non-carrier; s.d., standard deviation; MMSE, Mini-Mental State Examination; ADAS-Cog, Alzheimer's Disease } \\
\text { Assessment Scale-cognition; PA, physical activity; MIE; moderate intensity exercise. } \\
\text { a Data missing for one participant. } \\
\text { b Whole brain volume expressed as a \% of total intracranial volume. }\end{array}$} \\
\hline
\end{tabular}




\section{Image acquisition}

Scanning was performed on a 3T Siemens (Siemens MedicalErlangen, Germany) Tim Trio scanner equipped with a 12-channel phased-array coil (Royal Melbourne Hospital, Melbourne, Australia). See supplementary material for more details.

\section{Study-specific template}

An unbiased, study-specific template was generated from the T1-MPRAGE nifti images for 25 healthy individuals (12 male, 13 female) who were part of the larger Aibl trial. The template was created using the Advanced Normalization Tools (ANTS) 1.9.v4 build template parallel script ${ }^{23}$ (see supplementary material). Using a group-wise averaged template has the advantage of preserving the distinct anatomical features of our older participants much more closely than with direct spatial normalisation to the standard SPM template MNI152.

\section{Image preprocessing}

Imaging data were transferred and processed on a Linux platform running MATLAB version 8.0 (The MathWorks, Natick, Massachusetts, USA). Preprocessing was performed with Statistical Parametric Mapping software (SPM8; Wellcome Trust Centre for Neuroimaging, UK) using a preprocessing pipeline as reported in Kerestes et al. ${ }^{24}$ In brief, preprocessing involved the following steps: slice timing correction, motion correction, co-registration of each participant's echo planar images to their respective coregistered $\mathrm{T}_{1}$ image, spatial normalisation of both the structural and functional images (re-sliced to $2 \mathrm{~mm}$ ) and spatial smoothing with a $6 \mathrm{~mm}$ full width half maximum Gaussian filter (see supplementary material for additional information).

\section{Functional connectivity analysis}

We performed a detailed multiple region of interest ('seed-based ROI') analysis. Our approach was based on the method of Margulies et al. ${ }^{16}$ Of note, Margulies and colleagues ${ }^{16}$ used 21 right hemisphere seeds to define the PCC and precuneus, 8 of which were used to characterise the PCC. In the current analysis, we adopted four of these seeds for our examination of functional connectivity of the dorsal and ventral PCC. The seeds were chosen because of their cytotectonic placement within clearly defined dorsal and ventral divisions of the PCC (see supplementary material for sulcal landmarks). Seeds were placed bilaterally in the dorsal and ventral PCC along a rostral-caudal axis with the following coordinates: dorsal PCC (rostral; $x= \pm 2, y=-36, z=35$ ); dorsal PCC (caudal; $x= \pm 2, y=-47, z=35$ ); ventral PCC (rostral; $x= \pm 1, y=-67, z=22$ ); ventral PCC (caudal; $x= \pm 2, y=-59, z=11$ ) (Fig. 1). In addition to our signals of interest, we derived estimates of white matter, cerebrospinal fluid (CSF) and global signal fluctuations for each participant by segmenting each participant's anatomical scan. Grey matter, white matter and CSF tissue maps were thresholded to ensure 50\% tissue probability and binarised to create three nuisance variable masks (white matter, CSF and a whole brain mask). Signals were then extracted for each seed region and nuisance mask by calculating the mean ROI value across the times series for each participant.

Functional connectivity maps were estimated for each PCC region for each participant by including the PCC time series and nuisance signals as predictors of interest and no interest, respectively, in whole brain, linear regression analyses in SPM8, conduced separately for each hemisphere. A high-pass filter of $128 \mathrm{~s}$ was used to remove low-frequency drifts. Each of the three nuisance covariates were fully orthogonalised and then removed from each seed's time series along with six head-motion parameters ( 3 translation, 3 rotation estimates) by liner regression, resulting in a general linear model that comprised four "noise-

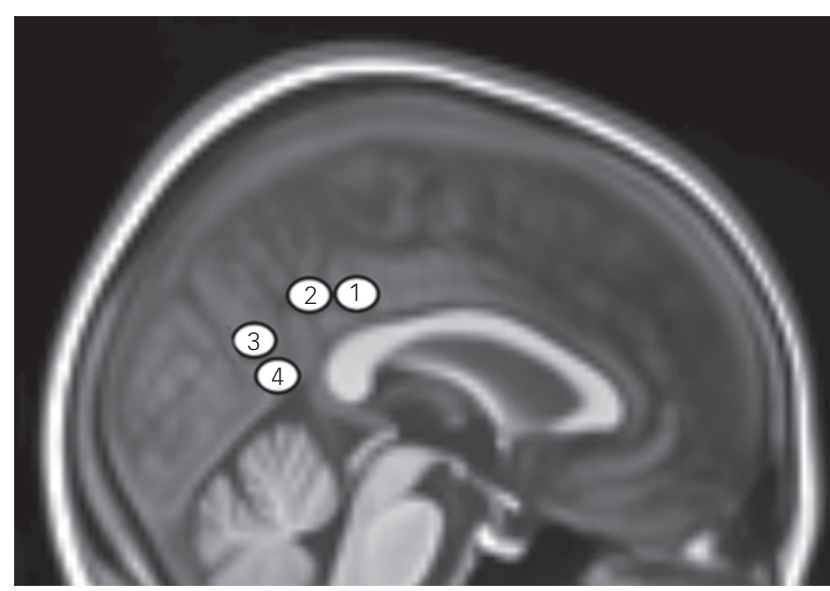

Fig. 1 Placement of the posterior cingulate cortex seed regions of interest on a $2 \mathrm{~mm}$ resolution anatomical study-specific template.

The study-specific template is registered to Montreal Neurological Institute space. Seed placement reflects the cytotectonic organization of the posterior cingulate cortex (PCC) where seeds 1 and 2 correspond to the dorsal PCC and seeds 3 and 4, the ventral PCC.

cleaned' seeds and nine nuisance variables. Contrast images were generated for each participant by estimating the regression coefficient between all brain voxels and each region's time series respectively.

For second-level, between-group analyses, a random effects $2 \times 2$ (group (APOE $\varepsilon 4+$, APOE $\varepsilon 4-)) \times$ (hemisphere (left seed, right seed)) factorial design was used in SPM8. Within-group statistical maps were thresholded at a false discovery rate (FDR) of $P<0.05$ for the whole brain. As an additional control for residual head motion influences, the mean inter-scan motion (calculated as the mean displacement between volumes across the scan) for each participant was included as a covariate in the second-level analysis as reported in Van Dijk et al. ${ }^{25}$ In addition, to control for heterogeneity in cognitive and functional deficits across SMC individuals, we took a continuous approach through analysis of covariance by including the presence or absence of mild cognitive changes as a covariate in our second-level analysis. We note that including the presence or absence of mild cognitive changes in our second-level models did not change the results (see supplementary material Fig. DS1). Each model in our second-level analysis was also covaried for age and gender. Between-group statistical maps were thresholded using a $P<0.001$ cluster-wise corrected threshold determined using a permutation-based framework (1000 permutations with a cluster-forming threshold of $P<0.001)$ as implemented in the REST toolbox. ${ }^{26}$ The minimum estimated cluster-sizes for significant connectivity with each seed were: dorsal PCC (rostral), 26 voxels; dorsal PCC (caudal), 25 voxels; ventral PCC (rostral), 26 voxels; ventral PCC (caudal), 18 voxels. See supplementary material for details on how cluster sizes were calculated.

\section{Brain-behaviour correlations}

To examine the relationship between PCC functional connectivity and measures of baseline physical activity in the APOE $\varepsilon 4+$ group, we performed a multiple linear regression analysis between two different measures of physical activity, one measure of endurance fitness, and connectivity of the four seed region connectivity maps. These measurements were: (a) total minutes of regular moderatevigorous intensity (SCI); ${ }^{27}$ (b) the 6 -min walk test, a validated, objective measure of cardiovascular endurance, ${ }^{28}$ measured by the maximum distance walked in a $20 \mathrm{~m}$ measured course; and (c) total number of steps taken per week, as objectively measured by a 
pedometer (Digi-Walker SW-200, Yamax, Tokyo, Japan) adjusted for time-on and time-off (e.g. when type of physical activity does not permit wearing the pedometer such as swimming). Results were displayed at $P<0.01$ cluster-wise corrected using a permutationbased framework (1000 permutations with a cluster-forming threshold of $P<0.01$ ) as implemented in the REST toolbox ${ }^{26}$ (see supplementary material for additional information).

\section{Results}

\section{Dorsal posterior cingulate (rostral) region}

The dorsal rostral PCC seed region in both groups showed significant functional connectivity with the middle and superior frontal gyrus, the anterior cingulate cortex, the angular gyrus region of the inferior parietal cortex, the middle and inferior temporal gyrus (Table 2). With regard to between-group differences, APOE $\varepsilon 4+$ individuals demonstrated significantly greater connectivity between the dorsal rostral PCC and the left middle frontal gyrus and right ventromedial prefrontal cortex (Fig. 2A, left and middle panels; Table 2). In contrast, APOE $\varepsilon 4$ individuals demonstrated increased connectivity with the right parahippocampal gyrus compared with APOE $\varepsilon 4+$ individuals (Fig. 2A, right panel; Table 2).

\section{Dorsal posterior cingulate (caudal) region}

The dorsal caudal PCC seed region in both groups showed significant functional connectivity with the middle frontal gyrus, anterior cingulate cortex as well as lateral occipital cortex and middle temporal gyrus (Table 2). APOE $\varepsilon 4+$ individuals showed significantly increased connectivity between the dorsal caudal seed and the right middle frontal gyrus and a region of the left precentral gyrus extending to the left middle frontal gyrus, compared with APOE $\varepsilon 4$ - individuals (Fig. $2 \mathrm{~B}$, right panel; Table 2). APOE $\varepsilon 4+$ individuals also showed increased connectivity with the left lateral occipital cortex (Fig. 2B, left panel; Table 2). APOE $\varepsilon 4$ - individuals did not show significantly greater connectivity with the dorsal caudal seed for any brain region.

\section{Ventral posterior cingulate (rostral) region}

The ventral rostral seed region in both groups showed significant functional connectivity with the precuneus and supracalcarine cortex, ventromedial frontal cortex and occipital cortex (Table 2). Compared with APOE $\varepsilon 4-$ individuals, APOE $\varepsilon 4+$ individuals showed significantly increased connectivity with a region of the left supplementary motor area (SMA) extending into the right SMA (Fig. 2C, middle panel; Table 2). There were no brain regions showing increased functional connectivity with the ventral rostral PCC seed in APOE $\varepsilon 4$ - individuals, compared with APOE $\varepsilon 4+$ individuals.

\section{Ventral posterior cingulate (caudal) region}

The ventral caudal seed region in both groups showed significant functional connectivity with the precuneus and intracalcarine cortex, middle frontal gyrus, supramarginal gyrus, central opercular cortex and inferior temporal gyrus (Table 2). Compared with APOE $\varepsilon 4-$ individuals, APOE $\varepsilon 4+$ individuals showed significantly increased connectivity with the right lateral occipital cortex and right inferior temporal gyrus (Fig. 2C, left panel; Table 2). In contrast, APOE $\varepsilon 4$ - individuals showed significantly increased connectivity compared with APOE $\varepsilon 4+$ individuals with the left middle frontal gyrus (Fig. 2C, right panel; Table 2).

\section{Brain-behaviour correlations}

In APOE $\varepsilon 4+$ individuals, a significant positive correlation was found between the distance covered in the 6 min walk test (higher endurance fitness) and connectivity between the ventral rostral PCC seed and the SMA $(x=-4, y=-16, z=56$; cluster size $=191$; $Z$ score 4.38; Fig. 3, centre and right upper panel). In addition, a significant positive correlation was found between the number of steps taken per week (higher habitual physical activity) and connectivity between the ventral rostral PCC seed and SMA, $(x=2, y=-16, z=54$; cluster size $=159, Z$ score 3.90; Fig. 3, centre and right lower panel). To delineate whether the above correlations were due to a general effect of physical activity or specific to APOE $\varepsilon 4$ risk, correlations were run for the two physical activity and one fitness measures in APOE $\varepsilon 4$ - individuals. There were no significant correlations with SMA connectivity for any of the PCC seeds, for any of the three measures in APOE $\varepsilon 4$ - individuals.

\section{Discussion}

This is the first study to systematically examine dorsal and ventral PCC connectivity in APOE $\varepsilon 4$ carriers and non-carriers in individuals at increased risk of cognitive decline and progression to Alzheimer's disease. Our results are partially consistent with previous findings of altered PCC connectivity in healthy APOE $\varepsilon 4$ carriers $^{2,11,29}$ but show for the first time, distinct alterations in the connectivity of dorsal and ventral divisions of the PCC in APOE $\varepsilon 4$ carriers at risk of Alzheimer's disease. Furthermore, our study adds to prior imaging findings by showing that increased ventral PCC connectivity with the SMA in APOE $\varepsilon 4+$ individuals is associated with physical endurance and habitual physical activity. This effect was specific to the ventral PCC.

The functional connectivity patterns of the PCC described in our study are largely consistent with the findings of Margulies et $a l,{ }^{16}$ showing some similarities but also distinct differences in the connectivity profiles of dorsal and ventral PCC. Specifically, across both groups, ventral posterior cingulate seeds showed connectivity with DMN regions and had greater connectivity with medial temporal lobe regions including the hippocampus, parahippocampal gyrus and amygdala. In contrast, dorsal posterior cingulate regions showed a pattern of connectivity that spanned different networks including parts of the frontoparietal network (dorsal/anterior prefrontal regions, rostral anterior cingulate), as well as areas implicated in the memory formation network (lateral and medial temporal regions). These results, which are also consistent with underlying cortico-cortical connections of the PCC in humans ${ }^{30}$ and monkeys, ${ }^{31}$ further validate the use of resting-state blood oxygen level dependent signal correlations for mapping brain connectivity, ${ }^{16}$ and the potential use of this technique for biomarker identification in Alzheimer's disease.

The most significant finding of the current study was an increase in functional connectivity of the rostral and caudal dorsal PCC with the middle frontal gyrus/dorsolateral prefrontal cortex (DLPFC) in APOE $\varepsilon 4+$ individuals. This was unexpected; we hypothesised that APOE $\varepsilon 4+$ individuals would show reduced functional connectivity with other resting-state networks, as previously shown in individuals at risk of Alzheimer's disease, ${ }^{8,32}$ and that this would be particularly evident for frontoparietal regions. This pattern of connectivity was more localised in APOE $\varepsilon 4-$ individuals compared with APOE $\varepsilon 4+$ individuals who showed more diffuse dorsal PCC connectivity with dorsal prefrontal regions. Although initially somewhat counterintuitive, given the conceptualisation of the DMN being anticorrelated with dorsal attention network regions, ${ }^{7,13}$ this relationship has been 


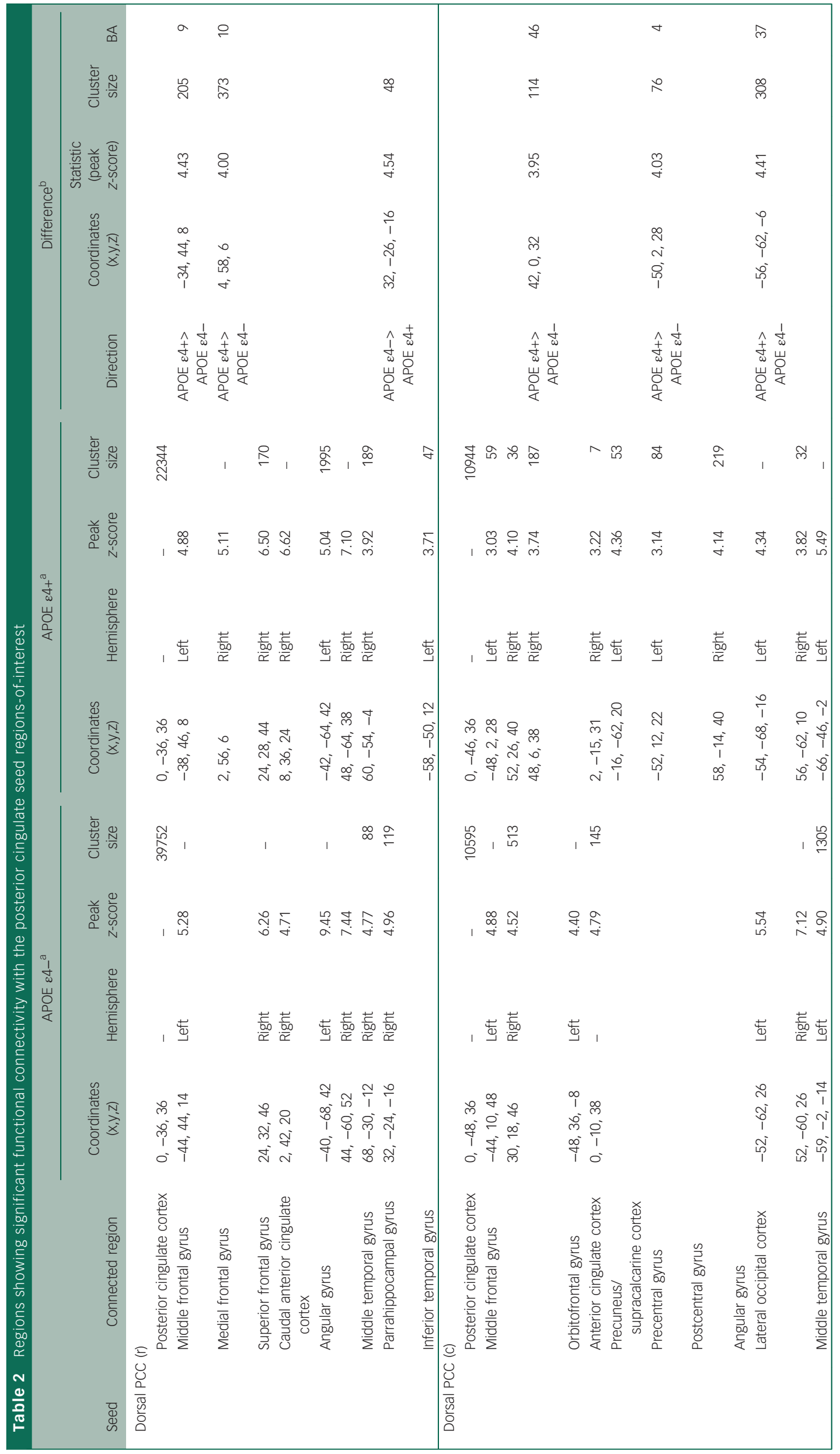




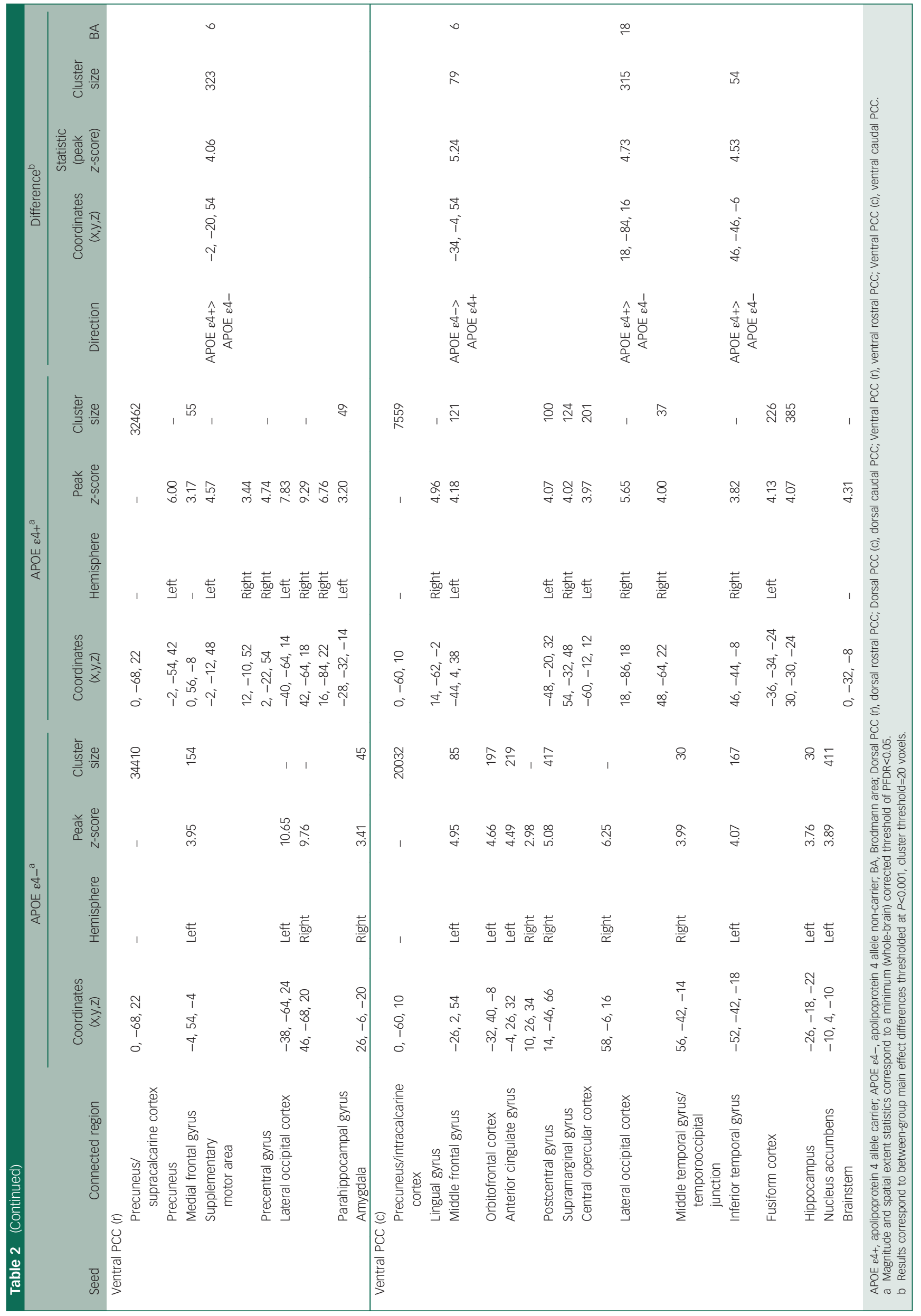




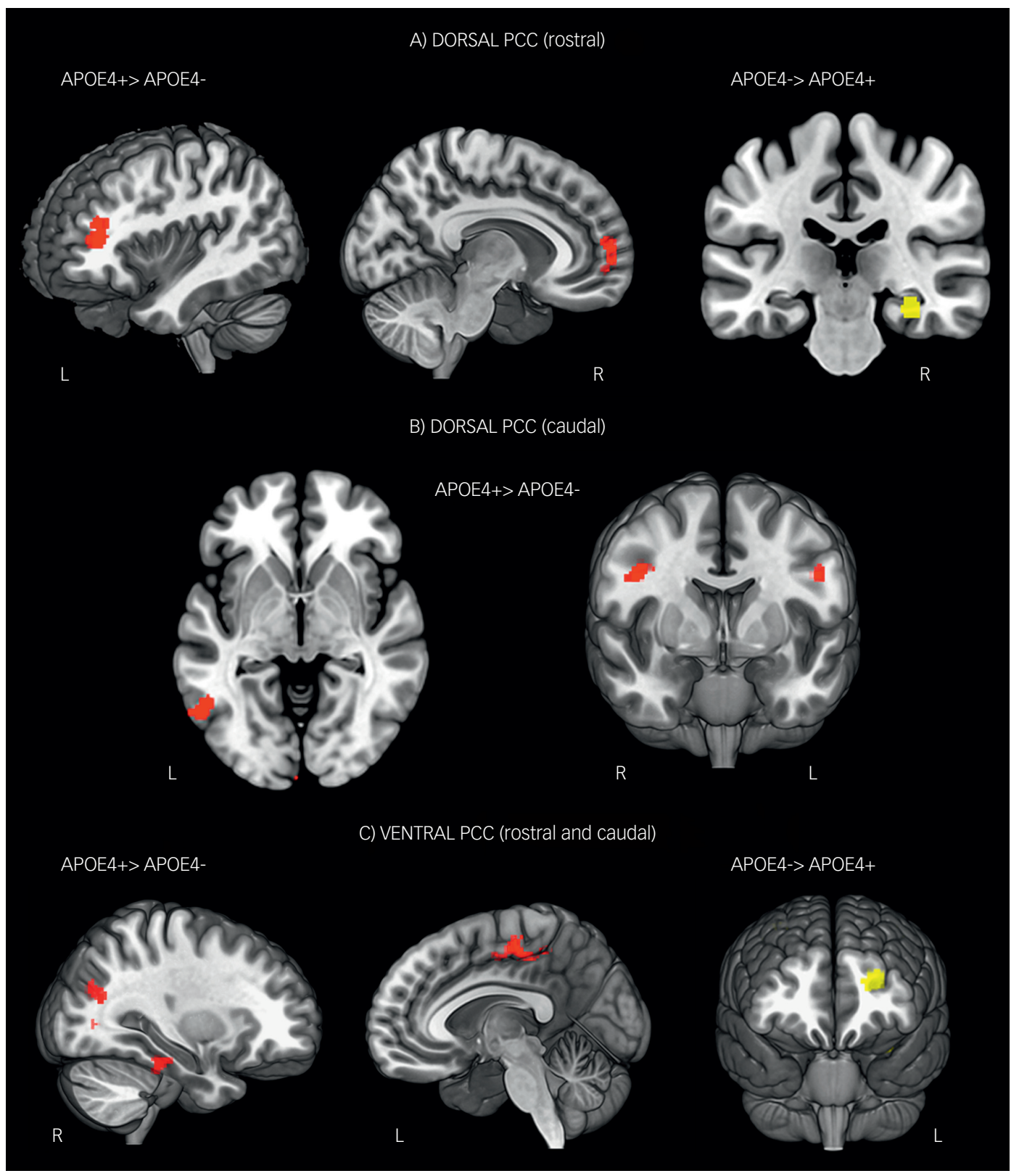

Fig. 2 Z-score maps showing significant between-group differences in functional connectivity of the dorsal (A and B) and ventral (C) posterior cingulate cortex.

Red=areas where connectivity was significantly greater in APOE $\varepsilon 4+$ individuals and yellow=areas where connectivity was significantly greater in APOE $\varepsilon 4-$ individuals. Results are shown at $P<0.001$, cluster-wise corrected.

shown most consistently during demanding cognitive tasks when external attentional requirements are high.

Recent literature suggests that although the ventral PCC is connected with DMN regions and anticorrelated with lateral prefrontal cortex regions in a tightly coupled way, the dorsal PCC shows a more transient pattern of connectivity with other intrinsic resting-state networks. ${ }^{17}$ Because this is the first study to examine connectivity of dorsal and ventral subdivisions of the PCC in individuals at increased risk of Alzheimer's disease, direct comparisons with other studies are not possible. There is, however, increasing evidence in the literature for increased PCC functional connectivity with lateral prefrontal cortex in individuals at risk of Alzheimer's disease, including older individuals with mild cognitive impairment, ${ }^{33}$ as well as cognitively healthy older APOE $\varepsilon 4$ carriers with $^{2}$ and without ${ }^{29}$ a family history of Alzheimer's disease.
The DLPFC is implicated in higher-order cognitive processes and integrated connectivity in dorsal attention and frontoparietal networks is important for sustained attention during periods of cognitive rest. ${ }^{34}$ Increased connectivity between the dorsal PCC and DLPFC in APOE $\varepsilon 4+$ individuals could therefore reflect compensatory recruitment of prefrontal regions necessary for executive and organisational functions. This may occur, for example, if there is disconnectivity in other cortical networks. In support of this view, and our hypothesis, we found reduced dorsal PCC right hippocampal connectivity in APOE $\varepsilon 4+$ individuals. Integrated connectivity between these two regions is thought to be important for episodic memory processes and several studies in SMC, mild cognitive impairment and Alzheimer's disease have reported disrupted PCC-hippocampal connectivity. ${ }^{8,13,33}$ Considered together, the increased dorsal PCC-DLPFC connectivity and reduced dorsal PCC-hippocampal connectivity we observed in 

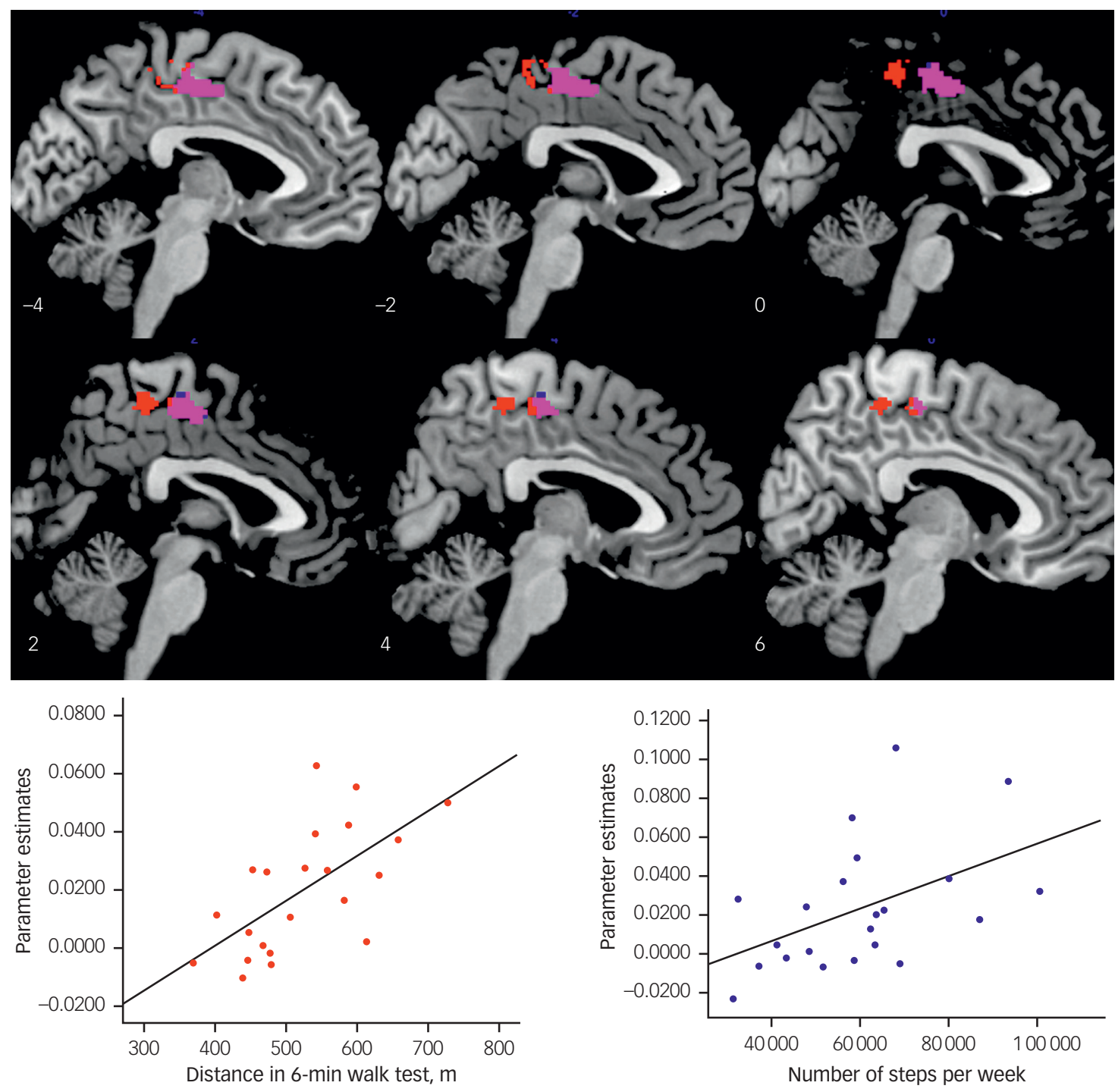

Fig. 3 Relationship between baseline physical activity and ventral (rostral) connectivity in APOE $\varepsilon 4+$ individuals.

Sagittal slices showing the region of supplementary motor area (SMA) that correlated with distance travelled in a 6-minute walk test (red) and total number of steps per week (blue). Violet=overlap between the two independent measures. Right (upper): scatterplot showing the significant association between 6-minute walk test scores ventral posterior cingulate cortex (PCC) connectivity strength with the SMA, $R=0.71, P=0.001$. Right (lower): scatterplot showing the significant association between total number of steps per week and ventral PCC connectivity strength with the SMA, $R=0.64, P=0.001$

APOE $\varepsilon 4+$ individuals may reflect a compensatory response to mediate frontal executive functions, as a result of a disconnection in dorsal PCC-hippocampal connectivity. Investigating the relationship between increased resting-state connectivity of DMN regions and how this relates to compensatory neural activity of these same regions during cognitive tasks in people at risk of Alzheimer's disease will remain important questions in future research.

We found an increase in ventral PCC-bilateral SMA functional connectivity in APOE $\varepsilon 4+$ individuals who positively correlated with both physical endurance and habitual physical activity. To our knowledge, this is the first study to report such an association. It is well established that regular physical activity significantly decreases the rate of cognitive decline in older adults, ${ }^{35,36}$ and several RCTs have shown that physical activity has a positive effect on cognition in healthy controls, individuals with SMC, mild cognitive impairment and patients with dementia. ${ }^{37,38}$ These effects may be mediated through neural plasticity of cortical networks that are associated with age-related dysfunction. The SMA is part of a sensorimotor network that is implicated in motor planning and selection, and studies have shown that this region is recruited during complex, finger tapping motor tasks in older adults to a greater degree than age-matched young individuals. ${ }^{39}$

A previously published RCT of aerobic exercise reported widespread increased functional connectivity of the PCC with DMN regions and frontal executive network regions in healthy older controls, following 12 months of aerobic (i.e. walking) exercise. ${ }^{19}$ Although direct comparisons to the Voss et al's study cannot be made, due to the cross-sectional nature of our study design, it is likely that our results reflect increased efficiency of neural network connectivity following physical activity, that appears to be modulated by genetic risk. Alternatively, increased connectivity between the highly interconnected ventral PCC and SMA may also simply reflect ongoing neural circuit changes that are occurring as a result of carrying the APOE $4 \varepsilon 4$ allele and being at elevated risk of cognitive decline. Our findings point to the importance of future longitudinal intervention studies that assess the effects of long-term physical activity on functional connectivity of brain networks and the extent to which this may alter the 
course of disease progression in individuals at risk of Alzheimer's disease. If such an effect of physical activity can be confirmed and replicated, this will have important clinical implications for treatment interventions in adults at risk of cognitive decline and Alzheimer's disease. Adopting and implementing physical activity programmes designed for older adults at risk of Alzheimer's disease, for example, when used in combination with cognitive interventions, may help delay the onset of clinical symptoms and the progression of cognitive impairment.

There are limitations of the present study. The participants recruited into our study were highly educated, predominantly White volunteers living in urban parts of Victoria. As such, the generalisability of our results is restricted. The lack of a control group of non-memory complaining healthy older adults imposes some restrictions on the validity and generalisability of the results. This limitation was, however, addressed by using an age-matched group and statistical method of regression to control for age in all of our analyses, so any between-group differences are likely not attributable to the normal aging process. In addition, our findings of PCC connectivity alterations were predominantly found in APOE e4 carriers. Given the much smaller sample size of APOE $\varepsilon 4$ carriers compared with non-carriers in our study, our findings need to be interpreted carefully and replicated in a bigger sample.

In conclusion, our systematic examination of dorsal and ventral PCC connectivity in individuals at increased risk of Alzheimer's disease show evidence of distinct alterations in functional connectivity of both dorsal and ventral PCC cortical circuits in APOE $\varepsilon 4$ carriers. These findings point to the importance of systematically examining dorsal and ventral divisions of the PCC in future studies. In addition, our finding of an association between physical activity and PCC connectivity in APOE $\varepsilon 4+$ individuals at increased risk of Alzheimer's disease provides further neurobiological evidence for the potential protective effect of physical activity in older adults at risk for cognitive decline. Future validation of these findings by longitudinal RCTs will have important implications for physical activity-based interventions that may slow down cognitive decline in individuals at risk of Alzheimer's disease.

Rebecca Kerestes, PhD, Department of Radiology, The Royal Melbourne Hospital, University of Melbourne, Parkville, Australia and Department of Psychiatry, University of Pittsburgh School of Medicine, Pittsburgh, Pennsylvania, USA; Pramit M. Phal, MBBS, FRANZCR, Chris Steward, PhD, Bradford A. Moffat, PhD, and Simon Salinas MSC (Eng), all at Department of Radiology, The Royal Melbourne Hospital, University of Melbourne, Parkville, Australia; Kay L. Cox, PhD, School of Medicine and

Pharmacology, University of Western Australia, Perth, Australia; Kathryn A. Ellis, PhD, Academic Unit for Psychiatry of Old Age, St. Vincent's Health, Department of Psychiatry, University of Melbourne, Melbourne, Australia; Elizabeth V. Cyarto, PhD National Ageing Research Institute, University of Melbourne, Parkville, Australia; David Ames, MBBS, FRANZC, FRCPsych, Academic Unit for Psychiatry of Old Age, St Vincent's Health, Department of Psychiatry, University of Melbourne, Melbourne, Australia and National Ageing Research Institute, University of Melbourne, Parkville, Australia; Ralph N. Martins, PhD, School of Medical Sciences, Edith Cowan University, Perth, Australia; Colin L. Masters, MD, The Florey Institute of Neuroscience and Mental Health, University of Melbourne, Melbourne, Australia; Christopher C. Rowe $M D$, FRACP, Department of Nuclear Medicine and Centre for PET, Austin Health, Heidelberg, Australia; Matthew J. Sharman, PhD, School of Health Sciences, University of Tasmania, Hobart, Australia; Olivier Salvado, PhD, Commonwealth Scientific Industrial Research Organization Preventative Health National Research Flagship, Australian e-Health Research Centre, Brisbane, Australia; Cassandra Szoeke, MBBS, FRACP, PhD, Michelle Lai, MBBS, MPH, Mmed, National Ageing Research Institute, University of Melbourne, Parkville, Australia; Nicola T. Lautenschlager, MD, Academic Unit for Psychiatry of Old Age, St. Vincent's Health, Department of Psychiatry, University of Melbourne, Melbourne, Australia and North Western Mental Health, Melbourne Health, Melbourne, Australia; Patricia M. Desmond, MD, Department of Radiology, The Royal Melbourne Hospital, University of Melbourne, Parkville, Australia

Correspondence: R. Kerestes, Department of Psychiatry, University of Pittsburgh School of Medicine, Pittsburgh, Pennsylvania, USA. Email: kerestesrv@upmc.edu

First received 21 Jun 2015, final revision 21 Aug 2015, accepted 16 Oct 2015

\section{Funding}

This work is supported by a project grant from the Australian National Health and Medical Research Council awarded to N.T.L., P.M.D., K.C., D.A., C.S., K.A.E., P.M.P., and C.M. (ID: 1005492)

\section{Acknowledgements}

We thank all the participants and our research staff for contributing to the study. We thank AIBL (www.aibl.csiro.au) for their support.

\section{References}

1 Bertram L, McQueen MB, Mullin K, Blacker D, Tanzi RE. Systematic meta-analyses of Alzheimer disease genetic association studies: the AlzGene database. Nat Genet 2007; 39: 17-23.

2 Fleisher AS, Sherzai A, Taylor C, Langbaum JB, Chen K, Buxton RB. Resting-state BOLD networks versus task-associated functional MRI for distinguishing Alzheimer's disease risk groups. Neuroimage 2009; 47: 1678-90.

3 Filippini N, MacIntosh BJ, Hough MG, Goodwin GM, Frisoni GB, Smith SM, et al. Distinct patterns of brain activity in young carriers of the APOE-epsilon4 allele. Proc Natl Acad Sci U S A 2009; 106: 7209-14.

4 Machulda MM, Jones DT, Vemuri P, McDade E, Avula R, Przybelski S, et al. Effect of APOE $\varepsilon 4$ status on intrinsic network connectivity in cognitively normal elderly subjects. Arch Neurol 2011; 68: 1131-6.

5 Fornito A, Bullmore ET. What can spontaneous fluctuations of the blood oxygenation-level-dependent signal tell us about psychiatric disorders? Curr Opin Psychiatry 2010; 23: 239-49.

6 Raichle ME, MacLeod AM, Snyder AZ, Powers WJ, Gusnard DA, Shulman GL. A default mode of brain function. Proc Natl Acad Sci U S A 2001; 98: 676-82.

7 Buckner RL, Andrews-Hanna JR, Schacter DL. The brain's default network: anatomy, function, and relevance to disease. Ann N Y Acad Sci 2008; 1124: 1-38.

8 Sorg C, Riedl V, Muhlau M, Calhoun VD, Eichele T, Laer L, et al. Selective changes of resting-state networks in individuals at risk for Alzheimer's disease. Proc Natl Acad Sci U S A 2007; 104: 18760-5.

9 Petrella JR, Sheldon FC, Prince SE, Calhoun VD, Doraiswamy PM. Default mode network connectivity in stable vs progressive mild cognitive impairment. Neurology 2011; 76: 511-7.

10 Hafkemeijer A, Altmann-Schneider I, Oleksik AM, van de Wiel L, Middelkoop HA, van Buchem MA, et al. Increased functional connectivity and brain atrophy in elderly with subjective memory complaints. Brain Connect 2013; 3: 353-62.

11 Sheline YI, Morris JC, Snyder AZ, Price JL, Yan Z, D’Angelo G, et al. APOE4 allele disrupts resting state fMRI connectivity in the absence of amyloid plaques or decreased CSF Aß42. J Neurosci 2010; 30: 17035-40.

12 Minoshima S, Giordani B, Berent S, Frey KA, Foster NL, Kuhl DE. Metabolic reduction in the posterior cingulate cortex in very early Alzheimer's disease. Ann Neurol 1997; 42: 85-94.

13 Greicius MD, Srivastava G, Reiss AL, Menon V. Default-mode network activity distinguishes Alzheimer's disease from healthy aging: evidence from functional MRI. Proc Natl Acad Sci U S A 2004; 101: 4637-42.

14 Buckner RL, Snyder AZ, Shannon BJ, LaRossa G, Sachs R, Fotenos AF, et al. Molecular, structural, and functional characterization of Alzheimer's disease: evidence for a relationship between default activity, amyloid, and memory. J Neurosci 2005; 25: 7709-17.

15 Leech R, Sharp DJ. The role of the posterior cingulate cortex in cognition and disease. Brain 2014; 137: 12-32.

16 Margulies DS, Vincent JL, Kelly C, Lohmann G, Uddin LQ, Biswal BB, et al. Precuneus shares intrinsic functional architecture in humans and monkeys. Proc Natl Acad Sci U S A 2009; 106: 20069-74.

17 Leech R, Braga R, Sharp DJ. Echoes of the brain within the posterior cingulate cortex. J Neurosci 2012; 32: 215-22

18 Cyarto EV, Lautenschlager NT, Desmond PM, Ames D, Szoeke C, Salvado O, et al. Protocol for a randomized controlled trial evaluating the effect of physical activity on delaying the progression of white matter changes on MRI in older adults with memory complaints and mild cognitive impairment: the AIBL Active trial. BMC Psychiatry 2012; 12: 167.

19 Voss MW, Prakash RS, Erickson KI, Basak C, Chaddock L, Kim JS, et al. Plasticity of brain networks in a randomized intervention trial of exercise training in older adults. Front Aging Neurosci 2010a; 2: e32.

20 Voss MW, Erickson $\mathrm{KI}$, Prakash RS, Chaddock L, Malkowski E, Alves $\mathrm{H}$, et al. Functional connectivity: a source of variance in the association between cardiorespiratory fitness and cognition? Neuropsychologia 2010; 48: 1394-406. 
21 Welsh KA, Butters N, Mohs RC, Beekly D, Edland S, Fillenbaum G, et al. The consortium to establish a registry for Alzheimer's disease (CERAD). Part V. A normative study of the neuropsychological battery. Neurology 1994; 44: 609-14.

22 Hixson JE, Vernier DT. Restriction isotyping of human apolipoprotein E by gene amplification and cleavage with Hhal. J Lipid Res 1990; 31: 545-8.

23 Avants BB, Tustison NJ, Song G, Cook PA, Klein A, Gee JC. A reproducible evaluation of ANTS similarity metric performance in brain image registration. Neuroimage 2011; 54: 2033-44.

24 Kerestes R, Harrison BJ, Dandash O, Stephanou K, Whittle S, Pujol J, et al. Specific functional connectivity alterations of the dorsal striatum in young people with depression. Neuroimage Clin 2014; 7: 266-72.

25 Van Dijk KR, Hedden T, Venkataraman A, Evans KC, Lazar SW, Buckner RL. Intrinsic functional connectivity as a tool for human connectomics: theory, properties, and optimization. J Neurophysiol 2010; 103: 297-321.

26 Song XW, Dong $Z Y$, Long $X Y$, Li SF, Zuo XN, Zhu CZ, et al. REST: a toolkit for resting-state functional magnetic resonance imaging data processing. PLOS One 2011; 6: e25031.

27 Marcus BH, Banspach SW, Lefebvre RC, Rossi JS, Carleton RA, Abrams DB. Using the stages of change model to increase the adoption of physical activity among community participants. Am J Health Promot 1992; 6: 424-9.

28 Rickli RE, Jones $\mathrm{CJ}$. The reliability and validity of a 6-minute walk test as a measure of physical endurance in older adults. JAPA 1998; 6: 363-75.

29 Yang AC, Huang CC, Liu ME, Liou YJ, Hong CJ, Lo MT, et al. The APOE $\varepsilon 4$ allele affects complexity and functional connectivity of resting brain activity in healthy adults. Hum Brain Mapp 2014; 35: 3238-48.

30 Hagmann $\mathrm{P}$, Cammoun L, Gigandet X, Meuli R, Honey CJ, Wedeen VJ, et al. Mapping the structural core of human cerebral cortex. PLOS Biol 2008; 6: e159.
31 Kobayashi Y, Amaral DG. Macaque monkey retrosplenial cortex: III. Cortical efferents. J Comp Neurol 2007; 502: 810-33.

32 Gili T, Cercignani M, Serra L, Perri R, Giove F, Maraviglia B, et al. Regional brain atrophy and functional disconnection across Alzheimer's disease evolution. J Neurol Neurosurg Psychiatry 2011; 82: 58-66.

33 Zhang HY, Wang SJ, Xing J, Liu B, Ma ZL, Yang M, et al. Detection of PCC functional connectivity characteristics in resting-state fMRI in mild Alzheimer's disease. Behav Brain Res 2009; 197: 103-8.

34 Vincent JL, Kahn I, Snyder AZ, Raichle ME, Buckner RL. Evidence for a frontoparietal control system revealed by intrinsic functional connectivity. J Neurophysiol 2008; 100: 3328-42.

35 Middleton LE, Barnes DE, Lui LY, Yaffe K. Physical activity over the life course and its association with cognitive performance and impairment in old age. J Am Geriatr Soc 2010; 58: 1322-6.

36 Scarmeas N, Luchsinger JA, Schupf N, Brickman AM, Cosentino S, Tang MX, et al. Physical activity, diet, and risk of Alzheimer disease. JAMA 2009; 302: 627-37.

37 Lautenschlager NT, Cox KL, Flicker L, Foster JK, van Bockxmeer FM, Xiao J, et al. Effect of physical activity on cognitive function in older adults at risk for Alzheimer disease: a randomized trial. JAMA 2008; 300: 1027-37.

38 Baker LD, Frank LL, Foster-Schubert K, Green PS, Wilkinson CW, McTiernan A et al. Effects of aerobic exercise on mild cognitive impairment: a controlled trial. Arch Neurol 2010; 67: 71-9.

39 Wu T, Hallett M. The influence of normal human ageing on automatic movements. J Physiol 2005; 562: 605-15. 